\title{
Relationship between the Floating Airborne Japanese Cedar Pollen Count and Inspired Antigen Dose in the Nasal Cavity
}

\author{
Hisayuki Kato, Kensei Naito, Chikasi Ito, \\ Syoji Saito, Kenji Takeuchi, Tamotsu Ogawa*, \\ Joji Ishii* \\ Department of Otolaryngology, Fujita Health University, School of Medicine, Aichi \\ *Nagoya City Public Health Research Institute, Aichi
}

\begin{abstract}
Determination of the relationship between the ambient airborne pollen count and inspired antigen dose in the human nose is important for to controlling the nasal symptoms of hay fever patients. We compared ambient floating Japanese cedar pollen counts with intranasal cedar pollen antigen doses in this study. Cedar pollen counts were made with two different devices, a Durham-type pollen sampler and a real-time pollen counter (KH-3000, Yamato Manufacturing Co., Ltd., Japan). Twelve healthy male adult volunteers remained seated next to the two devices airborne pollen counting with a nasal air sampler placed on each nostril for one hour during quiet nasal breathing on four randomly chosen days during the cedar pollinating season in 2003. Cry $\mathrm{j}$ 1 protein, one of the major allergens in Japanese cedar pollen was measured in the air collected by the nasal sampler by an enzyme-linked immunosorbent assay with anti-Cry $\mathrm{j} 1$ antibody. The intranasal cedar antigen dose was significantly correlated with the floating pollen count determined by both of the airborne pollen counting devices. Thus, it appears that the intranasal cedar antigen dose can be predicted from environmental airborne cedar pollen counts.
\end{abstract}

Key words : cedar pollen, cedar pollinosis, airborne pollen, pollen counts, intranasal antigen dose

\section{Introduction}

Since the population of patients with Japanese-cedar pollinosis in Japan has increased in recent decades, seasonal and daily information on airborne cedar pollen counts would be useful to large numbers of patients. The severity of nasal and ophthalmic symptoms varies directly with the floating cedar pollen count ${ }^{1)}$. Determination of the relationship between the ambient airborne pollen count and inspired antigen dose in the human nose is important for controlling nasal symptoms in patients with this seasonal nasal allergy. However, although investigations of airborne cedar pollen counts have been widely performed in Japan, the relationship between the airborne cedar pollen count and intranasal antigen dose is still unclear ${ }^{2}$. We therefore compared environmental airborne cedar pollen counts with intrana- sal cedar pollen antigen doses in this study.

\section{Materials and Methods}

As shown in Fig. 1, airborne cedar pollen counts during one hour, 1:00 pm to 2:00 pm, were made with a Durham-type pollen sampler and a real-time pollen counter (KH-3000 ; Yamato Manufacturing Co., Ltd. Japan). These two devices were placed on the roof of the first floor of Fujita Health University Hospital. Japanese-cedar pollen within a $1 \times 1 \mathrm{~cm}^{2}$ area was counted by the Durham-type pollen sampler, and it was counted per liter of air by the real-time pollen counter. The afternoon of the March 8, 14, 20, and 23, 2003 were randomly chosen for this study.

Twelve healthy male adult volunteers (mean age: $34.2 \pm 7.9$ years) consented to take part in this study and remained in the sitting position with quiet nasal 
breathing adjacent to the two airborne pollen sampling devices for the one hour period on each day. As shown in Fig. 2, the volunteers wore Oriental adult-nostril-size nasal samplers on their nostrils manufactured by Dr. E. Tobey of Sydney University, Australia. Two of the twelve volunteers participated in the study on the March 8, five on March 14, three on March 20, and two on March 23.

Cry $\mathrm{j} 1$, one of the major allergens of Japanese-cedar pollen, was extracted from the nasal sampler with phosphate-buffered solution. The amount of Cry $\mathrm{j} 1$ protein in the cedar pollen was measured by enzyme-linked immunosorbent assay using anti-Cry j 1 antibody ${ }^{3)}$.

We compared the airborne cedar pollen counts assessed by the two devices with the intranasal Cry $\mathrm{j} 1$ doses, and Pearson product-moment correlation coeffi- cients were calculated to analyze the significance of the correlations.

\section{Results}

The status of each volunteers, the Cry j 1 antigen doses inspired in to the nasal cavity, and floating airborne cedar pollen counts are shown in Table 1 . As shown in Fig. 3, the correlation between the environmental floating airborne cedar pollen count according to the Durham-type pollen sampler and the inspired intranasal Cry j 1 dose was significant $(r=0.955, p<$ 0.001). As shown in Fig. 4, the correlation between environmental floating airborne cedar pollen count according to the real-time pollen counter and the inspired intranasal Cry j 1 dose was also significant $(\mathrm{r}=0.913, \mathrm{p}<$ $0.001)$.

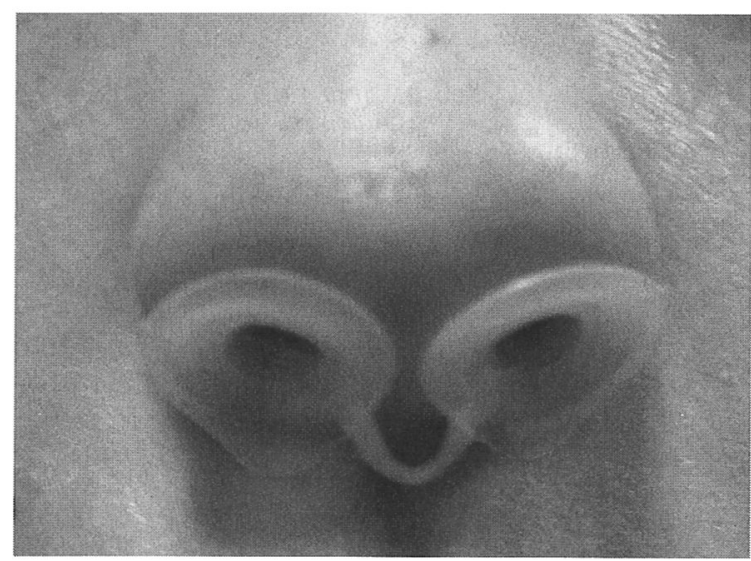

Fig. 2 A nasal sampler made of silicon (manufactured by Dr. E. Tobey of the University of Sydney, Australia).

Table 1 Volunteers status, Cry j 1 doses, and floating cedar pollen count

\begin{tabular}{ccccccccc}
\hline Case & age & height & weight & TV & date & Cry j 1 & Durham & KH-3000 \\
\hline 1 & 24 & 168 & 65 & 3729 & 8/Mar & 0.5 & 10 & 5 \\
2 & 25 & 172 & 78 & 3872 & 8/Mar & 0.5 & 10 & 5 \\
3 & 29 & 173 & 82 & 4212 & 14/Mar & $<0.25$ & 3 & 1 \\
4 & 26 & 177 & 78 & 4231 & 14/Mar & $<0.25$ & 3 & 1 \\
5 & 25 & 174 & 72 & 4210 & 14/Mar & $<0.25$ & 3 & 1 \\
6 & 27 & 166 & 62 & 3756 & 14/Mar & $<0.25$ & 3 & 1 \\
7 & 33 & 167 & 73 & 3892 & 14/Mar & $<0.25$ & 3 & 1 \\
8 & 32 & 162 & 68 & 3626 & 20/Mar & 2.0 & 49 & 12 \\
9 & 36 & 171 & 75 & 3924 & 20/Mar & 3.5 & 49 & 12 \\
10 & 28 & 169 & 70 & 4011 & 20/Mar & 4.0 & 49 & 12 \\
11 & 28 & 164 & 72 & 3787 & 23/Mar & $<0.25$ & 1 & 1 \\
12 & 31 & 168 & 71 & 3892 & 23/Mar & $<0.25$ & 1 & 1 \\
\hline
\end{tabular}

age : y.o., height : $\mathrm{cm}$, weight $\mathrm{Kg}$, TV (tidal volume) : $\mathrm{ml}$, Cry j $1: \mathrm{ng} / 5 \mathrm{ml}$

Durham : pollen/cm2, KH-3000 : pollen/1 


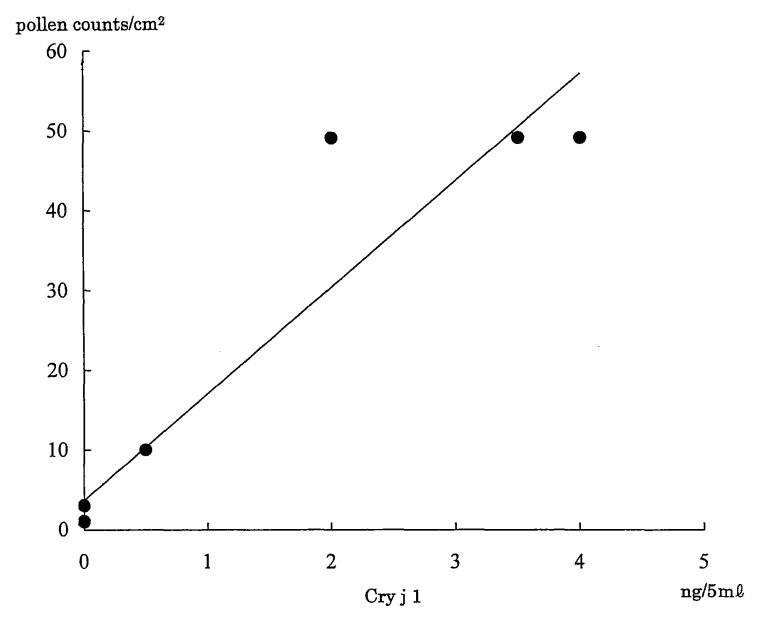

Fig. 3 Correlation between airborne cedar pollen count determined by a Durham-type pollen sampler and intranasal Cry j 1 dose $(r=0.955, p<0.001)$.

\section{Discussion}

The number of Japanese-cedar (Cryptomeria japonica) pollinosis patients in Japan has been increasing, and more than $10 \%$ of the population of the country now suffer from this seasonal nasal allergy ${ }^{1,4)}$. An increase in Japanese-cedar pollen count has been pointed out as the major cause of the increase in the number of patients, and the increase in pollen is due to promotion of cedar tree plantation for prompt industrial use under the direction of the Japanese government since Second World War ${ }^{1,5)}$, although environmental pollution and a westernized life style, including foods, and progress in social hygiene also may be other causes of this increase ${ }^{6,7)}$.

Floating cedar pollen counts vary both seasonally and daily ${ }^{8)}$, and since the severity of pollinosis symptoms depends on the ambient airborne cedar pollen count ${ }^{7,8}$, information on daily and yearly airborne cedar pollen counts might be beneficial to a great number of patients with cedar pollinosis. The degree of local exposure of the upper airway to cedar pollen antigen was found to affect sensitization and the severity of upper respiratory allergic reaction in animal experiments ${ }^{5}$, but, the relationship between floating cedar pollen count and inspired antigen dose in the human nose has remained unclear. In this study, we investigated this relationship by comparing ambient airborne cedar pollen counts and inspired Cry $\mathrm{j} 1$ doses in the human nasal cavity, and the result showed that the environmental airborne cedar pollen count was significantly correlated with inspired cedar

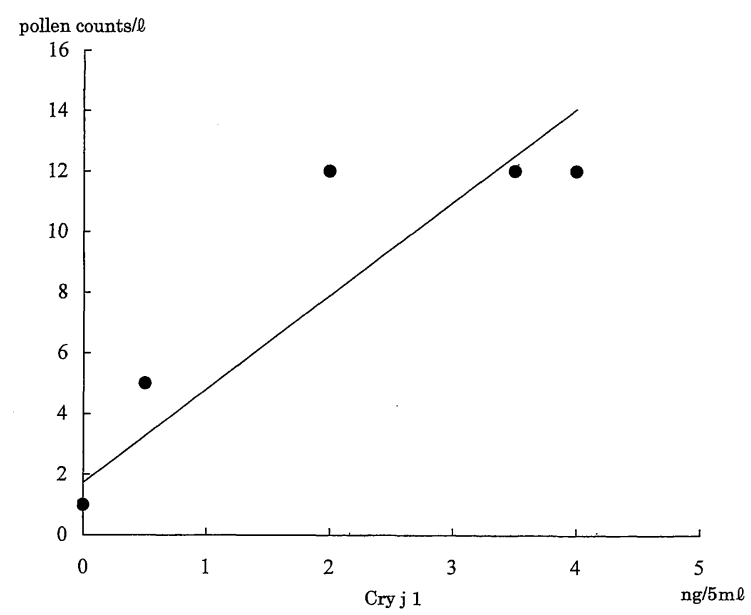

Fig. 4 Correlation between airborne cedar pollen count determined by a real-time pollen counter and intranasal Cry j 1 dose $(r=0.913, p<0.001)$.

pollen antigen dose in the adult human nose.

We used two types of airborne pollen samplers, a Durham-type pollen sampler and a real-time pollen counter. The former instrument collects floating pollen that falls naturally on to a slide glass inside it, and it is commonly used in Japan. The latter can measure real-time cedar and cypress pollen counts, and its use in Japan has recently increased, because it enables immediate determination of pollen counts. The real-time pollen sampler allows information on airborne cedar pollen to be instantaneously relayed to hay fever patients. Pollen counts measured with the real-time pollen counter have been reported to be significantly correlated with those measured by the Durham-type pollen sampler" ${ }^{9}$. Pollen counts measured by the Durham-type pollen sampler before the appearance of the real-time pollen counter in our area can thus be utilized based on the correlation equation of for the pollen counts measured by the two devices.

In our earlier study using the real-time pollen counter, the weather conditions that increased airborne cedar pollen during pollinating seasons were found to consist of high temperature, high wind velocity, and low humidity $^{9}$. Thus, the intranasal cedar antigen dose during the cedar pollinating season can be predicted from airborne pollen counts measured with the real-time pollen counter, and possibly from weather forecasts.

On the other hand, the reactivity of the nasal mucosa of patients increases during the pollination season and decreases off-season ${ }^{10)}$. This phenomenon is assumed 
to be due to nonspecific local hyperreactivity following repeated exposure to pollen ${ }^{11)}$. We also found that the variability in nasal patency of patients with cedar pollinosis might be related not only to the amount of floating pollen and nonspecific hypersensitivity of the nasal mucosa following environmental pollen exposure but to the endogenous circannual rhythm ${ }^{12)}$.

Further investigations are needed to determine the relationship between nasal symptoms in cedar pollinosis patients and intranasal antigen doses, including as modified by these exacerbating factors, and the efficacy of either preseasonal or seasonal oral antihistamine use in treating nasal symptoms during natural exposure to airborne aeroallergens.

\section{References}

1) Naito K, Ishii G, Ogawa $T$, et al : Specific $\operatorname{IgE}$ and IgG4 antibodies to Japanese cedar pollen and total $\operatorname{IgE}$ antibody in lumbermen. Aerobiologia $14: 321^{-}$ 324, 1998.

2 ) Saito Y : Airborne pollen survey of Cryptomeria japonica and cupressaceae in Yushima, Bunkyo-ku, Tokyo in 1996 and past 20 years (1977-1996). Jpn J Palynol 42 : 149-153, 1996. (in Japanese)

3 ) Yasueda H, Yui Y, Shimizu T, et al: Isolation and partial characterization of the major allergen from Japanese cedar (Cryptomeria japonica) pollen. J Clin Immunol 71: 77-86, 1983.

4) Imaoka K, Miyazawa H, Nishihara S, et al: Effect of pollen exposure on serum IgE and IgG antibody responses in Japanese cedar pollinosis patients. Allergol Int 45 : 159-162, 1996.

5 ) Sakurai K, Naito K, Ishii G, et al : Influence of local antigen exposure dose in the upper respiratory tract on sensitization with cedar pollen. Allergol Int 51: 9-12, 2002.

6 ) Saxson A, Zhang K, Takenaka $\mathrm{H}$, et al: The role of diesel exhaust in the allergic antibody response of the human airway. J Jpn Immunol Allergol Otolaryngol 14 : 9-11, 1996.

7 ) Naito K, Iwata S, Yokoyama N : Laryngeal symptoms in patients exposed to Japanese cedar pollen : allergic reactions and environmental pollution. Eur Arch Otorhinolaryngol 256 : 209-211, 1999.

8 ) Naito K, Ibata K, Ishihara M, et al: Seasonal variations of nasal resistance in allergic rhinitis and environmental pollen counts. Auris Nasus Larynx 20 : 19-29, 1993.

9 ) Ito C, Naito K, Saito S, et al : Pollen counts for Japanese cedar and cypress using a real-time pollen counter at Toyoake City, Aichi for 2001-2004. Jpn J Rhinol 43 : 380-384, 2004. (in Japanese)

10) Konno A, Togawa $K$, Nishihara $S$ : Seasonal variation of sensitivity of nasal mucosa in pollinosis. Arch Otorhinolaryngol 232 : 253-261, 1981.

11) Connell JT: Quantitative intranasal pollen challenges II. Effect of daily pollen challenge, environmental pollen exposure, and placebo challenge on the nasal membrane. J Allergy 41: 123-139, 1968.

12) Naito $K$, Ishihara $M$, Senoh $Y$, et al : Seasonal variations of nasal resistance in allergic rhinitis and environmental pollen counts II : Effrcacy of preseasonal therapy. Auris Nasus Larynx $20: 31-38$, 1993.

（2005年 3 月 23 日受稿， 2005 年 7 月 11 日受理）

Reprint and correspondence address :

Hisayuki Kato, M.D.

Department of Otolaryngology,

Fujita Health University, School of Medicine

1-98 Kutsukake Toyoake, Aichi 470-1192 Japan

Tel : +81-562-93-9291

Fax : +81-562-95-0566 